
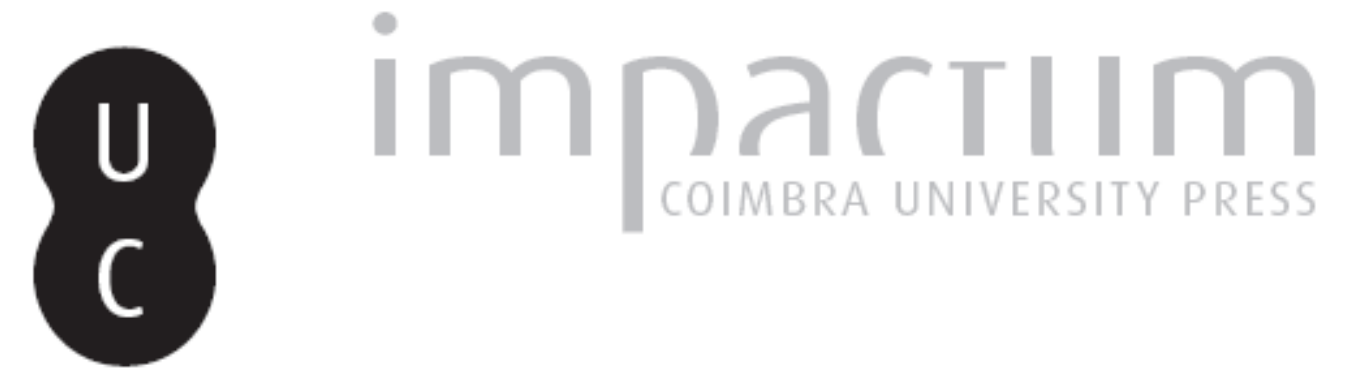

\title{
Chiusura causale della fisica e razionalità del tutto: alcune opzioni esegetiche sull'efficienza causale delle idee platoniche
}

Autor(es): $\quad$ Fronterotta, Francesco

Publicado por: Imprensa da Universidade de Coimbra

URL persistente:

URI:http://hdl.handle.net/10316.2/42209

DOI:

DOI:https://doi.org/10.14195/2183-4105_8_3

Accessed : $\quad$ 26-Apr-2023 13:17:58

A navegação consulta e descarregamento dos títulos inseridos nas Bibliotecas Digitais UC Digitalis, UC Pombalina e UC Impactum, pressupõem a aceitação plena e sem reservas dos Termos e Condições de Uso destas Bibliotecas Digitais, disponíveis em https://digitalis.uc.pt/pt-pt/termos.

Conforme exposto nos referidos Termos e Condições de Uso, o descarregamento de títulos de acesso restrito requer uma licença válida de autorização devendo o utilizador aceder ao(s) documento(s) a partir de um endereço de IP da instituição detentora da supramencionada licença.

Ao utilizador é apenas permitido o descarregamento para uso pessoal, pelo que o emprego do(s) título(s) descarregado(s) para outro fim, designadamente comercial, carece de autorização do respetivo autor ou editor da obra.

Na medida em que todas as obras da UC Digitalis se encontram protegidas pelo Código do Direito de Autor e Direitos Conexos e demais legislação aplicável, toda a cópia, parcial ou total, deste documento, nos casos em que é legalmente admitida, deverá conter ou fazer-se acompanhar por este aviso.

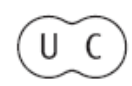




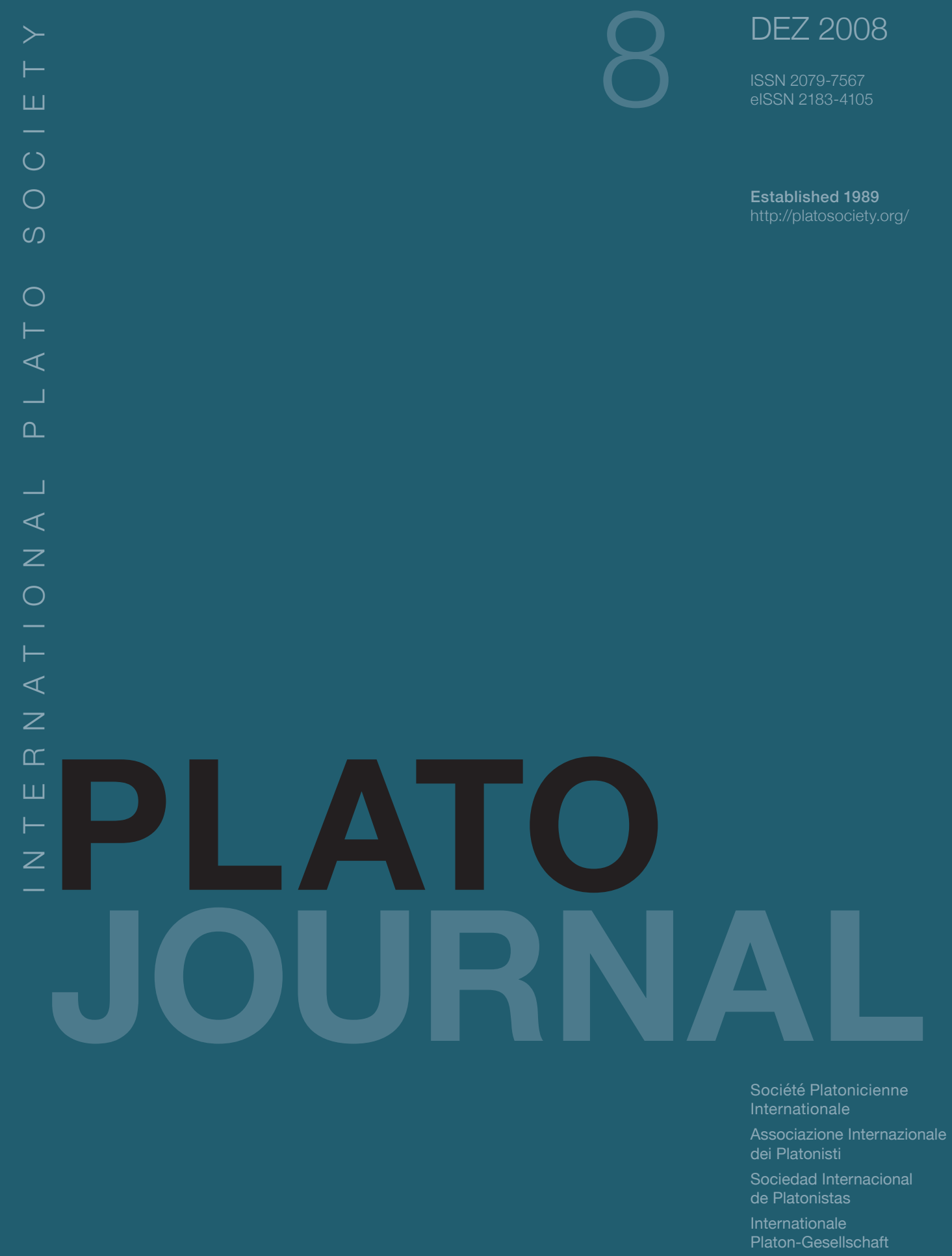




\section{CHIUSURA CAUSALE DELLA FISICA E RAZIONALITÀ DEL TUTTO : ALCUNE OPZIONI ESEGETICHE SULL'EFFICIENZA CAUSALE DELLE IDEE PLATONICHE}

Una tendenza interpretativa recente attribuisce alle idee platoniche una forma di causalità, rispetto alle cose sensibili, di natura produttiva o efficiente, cioè tale che le idee producono o generano nelle cose sensibili che ne partecipano la proprietà di cui sono le idee e di cui le cose partecipanti sono prive prima di tale partecipazione. Un certo numero di studi significativi negli ultimi decenni ha infatti via via contestato l'impostazione che al problema della causalità delle idee era stata data da Gregory Vlastos in un celebre articolo degli anni '60, che riconosceva agli intellegibili una forma di causalità esclusivamente formale e in certa misura paradigmatica (1). Personalmente, condivido largamente questa tendenza interpretativa, alla quale ho tentato io stesso di contribuire (2). Mi limito a ricordare qui una breve sequenza di argomenti e testimonianze testuali che, a mio avviso, confortano questo esito teorico: non entrerò però nel merito di una discussione di questi argomenti, perché assumerò qui, come ipotesi di lavoro, la loro validità, per concentrarmi piuttosto su alcune delle conseguenze che essi comportano.

Nel Simposio (211b2-5), Platone afferma che, sebbene le cose sensibili mutevoli e corruttibili partecipino dell'idea del bello, il bello in sé rimane tuttavia incorrotto

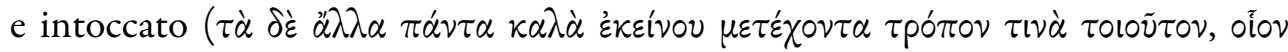

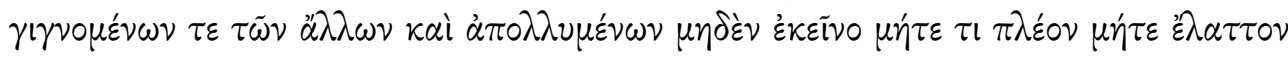
$\gamma^{\prime}(\gamma \nu \varepsilon \sigma \theta \alpha \iota \mu \eta \delta \dot{\varepsilon} \pi \alpha \dot{\alpha} \sigma \varepsilon\llcorner\nu \mu \eta \delta \dot{\varepsilon} \nu)$. Una simile, ovvia precisazione si rende necessaria solo a patto che, sussistendo una reale congiunzione fra la sfera sensibile e il

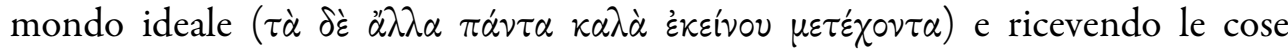
sensibili belle la propria bellezza direttamente dal bello in sé, le idee si trovino esposte al rischio di una contaminazione da parte delle cose sensibili, che le ponga

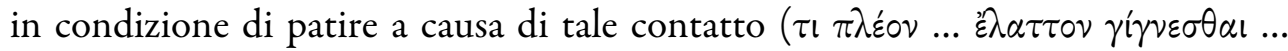

PLATO, The electronic Journal of the International Plato Society, n 8, 2008.

http://gramata.univ-paris1.fr/Plato

(c) All rights of reproduction of any form reserved. 
$\pi \dot{\alpha} \sigma \chi \varepsilon เ \nu$...): se così non fosse, perché Platone sentirebbe il bisogno di ribadire la natura incorruttibile dell'idea del bello? Ancora, nel Parmenide (129a3-6), in polemica con Zenone, Socrate introduce la sua teoria delle idee pronunciandosi in maniera esplicita sul significato che egli attribuisce al meccanismo della partecipazione: se esistono le idee della somiglianza e della dissomiglianza, è legittimo sostenere che ciò che partecipa dell'idea della somiglianza per questo

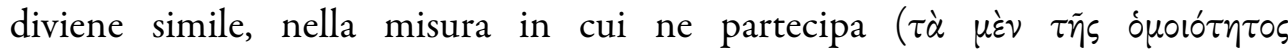

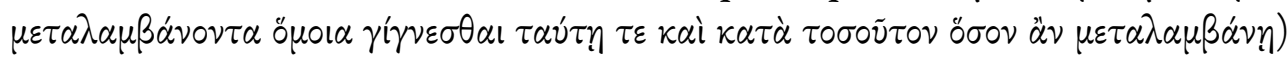
e ugualmente per quanto riguarda la dissomiglianza e tutti gli altri generi. Pare insomma che le idee non solo permettano al soggetto conoscente di identificare mentalmente e concettualmente l'essenza e le proprietà fisiche in possesso delle cose partecipanti, ma si configurino piuttosto come l'origine stessa e il principio metafisico dell'essenza e delle proprietà fisiche in possesso delle cose partecipanti, ossia del processo di acquisizione ( $\gamma^{\prime}(\gamma \nu \varepsilon \sigma \theta \alpha \mathrm{l})$, da parte delle cose partecipanti, di una certa essenza e di determinate proprietà fisiche.

Nel Fedone (100e5-6 e 101c2-9) Socrate procede a descrivere l'articolazione della

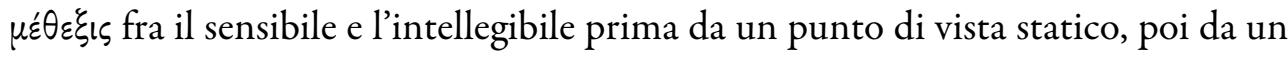
punto di vista dinamico. Ciò che è, per esempio, grande, lo è certamente in virtù della (partecipazione alla) grandezza in sé ( $\mu \varepsilon \gamma \varepsilon \dot{\varepsilon} \theta \varepsilon \mathrm{\tau} \tau \dot{\alpha} \mu \varepsilon \gamma \dot{\alpha} \lambda \alpha \mu \varepsilon \gamma \alpha \dot{\lambda} \lambda \alpha)$; ma ciò che, mutando il proprio stato, entra in possesso di una qualunque caratteristica, viene a partecipare dell'idea corrispondente a quella caratteristica: infatti, il divenire 'due' non dipende che dalla partecipazione alla dualità in sé e ciò che diviene 'due'

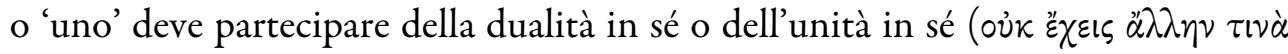

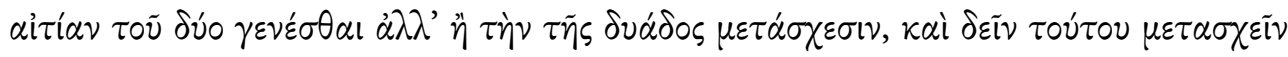

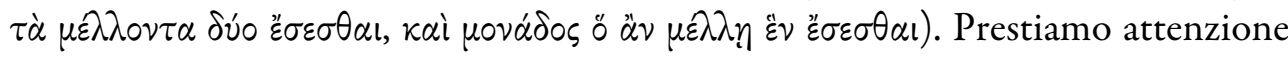
al momento dinamico della spiegazione di Socrate: se, per entrare in possesso di una qualunque caratteristica o per mutare il proprio stato, le cose sensibili devono partecipare delle idee corrispondenti a quella caratteristica o a quello stato, è allora necessario dedurne che le idee trasmettono effettivamente qualcosa che determina una differenza essenziale nelle cose sensibili e produce in esse una sostanziale modificazione. Prima di partecipare della grandezza in sé, un albero sensibile, per esempio, non è grande e non può essere definito 'grande'; se diviene grande, ciò significa necessariamente che l'albero viene a partecipare della

PLATO, The electronic Journal of the International Plato Society, n 8, 2008.

http://gramata.univ-paris1.fr/Plato

(c) All rights of reproduction of any form reserved. 
grandezza in sé (o viceversa: se viene a partecipare della grandezza in sé, l'albero sensibile diverrà necessariamente grande). Analogamente, se un ente sensibile è 'uomo' in virtù dell'idea di uomo, prima di partecipare dell'idea di uomo esso è, poniamo, pura materia informe e non può essere definito 'uomo'; attraverso la partecipazione, questa materia informe riceve dall'idea di uomo la struttura e la costituzione fisica che rispecchiano in forma materiale e sensibile l'essenza metafisica dell'idea di uomo e acquista conseguentemente il nome e la definizione di 'uomo'. Nell'ambito del rapporto partecipativo non si realizzano perciò semplicemente una classificazione concettuale o un'inferenza analitica che, a partire da alcune premesse logico-metafisiche, conducono al riconoscimento di una certa caratteristica o di uno stato fisico, ma si compiono effettivamente la trasmissione o la comunicazione reale e concreta di quella caratteristica o di quello stato fisico dalle idee alle cose empiriche.

Inoltre, al di là delle testimonianze dirette, è opportuno considerare un argomento filosofico di fondo. Ammettiamo pure per ipotesi che alle idee competa esclusivamente il potere di consentire al soggetto conoscente di riconoscere $\mathrm{e}$ di individuare le caratteristiche che le cose sensibili già possiedono $\mathrm{o}$ lo stato fisico in cui esse già si trovano: in questo caso, la partecipazione consisterebbe evidentemente, in senso (neo-)kantiano (3), in tale operazione mentale di 'riconoscimento' e di 'individuazione'. Ma come potrebbero le cose sensibili possedere di per sé determinate caratteristiche o trovarsi di per sé in uno stato fisico ben definito? Secondo Platone, le cose sensibili, considerate di per sé,

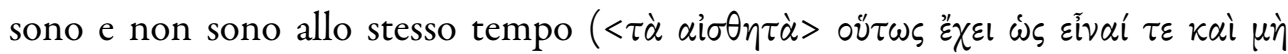

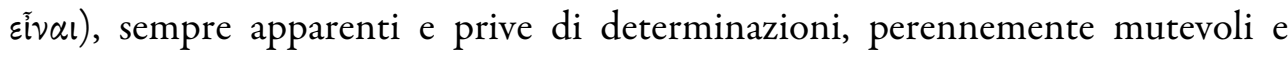

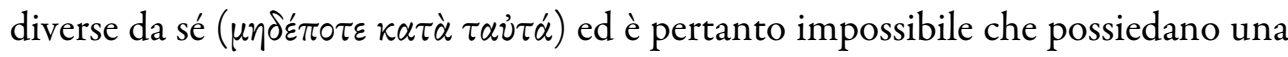
certa caratteristica o si trovino in un certo stato fisico, se non istantaneamente e per caso e divenendo subito altre e d'altra specie (cfr. per esempio Crat. 439d8-e2; Phaed. 74a9-e5; 78c6-79a5; Rep. V 478e7-479d1). Invece, per possedere una certa caratteristica o trovarsi in un certo stato fisico, nelle forme e nei modi solo parzialmente stabili e veri che sono loro consentiti, occorre che esse partecipino delle idee e ne ricevano le determinazioni per l'intera durata della relazione di partecipazione. Infatti, appena cessano di partecipare delle idee, le cose ricadono nella condizione loro propria e, immediatamente mutando e corrompendosi,

PLATO, The electronic Journal of the International Plato Society, n 8, 2008.

http://gramata.univ-paris1.fr/Plato

(c) All rights of reproduction of any form reserved. 
perdono ogni caratterizzazione specifica. È dunque in virtù della partecipazione alle idee che le cose sensibili entrano in possesso, benché in forma parziale e imperfetta, delle caratteristiche che alle idee appartengono in forma compiuta e perfetta.

Va considerato infine che il linguaggio che designa la causalità eidetica in Platone, fin dai dialoghi giovanili e particolarmente proprio nel Fedone, pare assai esplicito delle idee, infatti, non si dice solo che 'fanno' ( $\pi \circ \varepsilon \varepsilon i v)$ qualcosa, ma anche che si

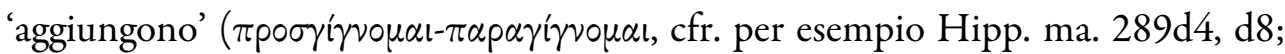

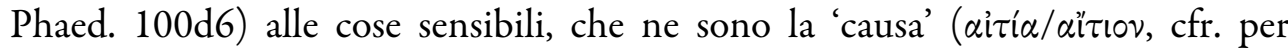
esempio Hipp. ma. 269e9, 297a1-4, a8, b1-2, c2; e ancora Phaed. 100b1-e3), il

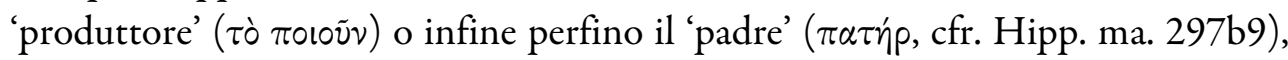
mentre il loro prodotto o effetto è qualificato come $\tau \grave{o} \gamma \iota \gamma \nu o ́ \mu \varepsilon v o v$ (cfr. Hipp. ma.

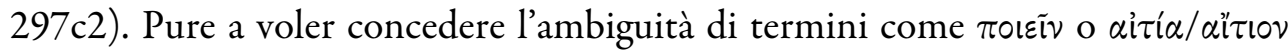
('cause' o 'ragioni'?), pare difficile intendere la relazione fra il padre e il figlio o fra il produttore e il prodotto generato diversamente da un rapporto di causalità che

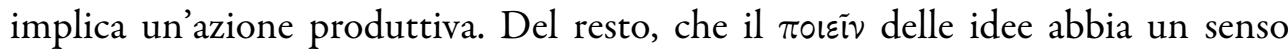
eminentemente produttivo e non il significato assai ampio e vario, e potenzialmente metaforico, che le lingue moderne attribuiscono al verbo 'fare', trova conferma nel fatto che, quando si trova in un contesto causale e assume un significato connesso all'azione causale, il $\pi \circ \varepsilon \tilde{\imath} \nu$ pare rimandare necessariamente a un processo produttivo o generativo. Possiamo citare in proposito Soph. $219 \mathrm{~b}$ (ma cfr. anche 265b), che contiene una delle più limpide esposizioni del principio che ispira la concezione platonica dello statuto delle cause e che governa il conseguente meccanismo della causalità: ovunque si constati un passaggio dal non essere all'essere, una generazione (e verosimilmente, per analogia, un passaggio dall'essere al non essere, una corruzione), bisogna supporre l'intervento di un

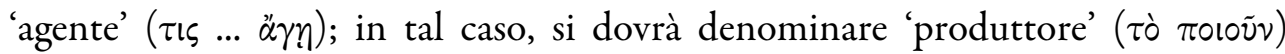

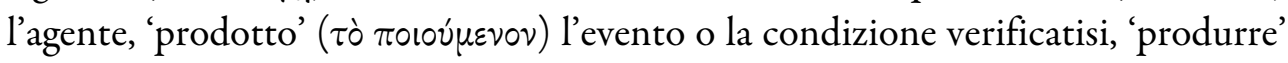

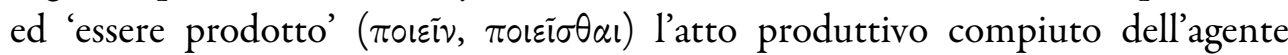
produttore e subito dal prodotto. È del tutto chiaro qui il riferimento a un genere di causalità che, a partire dalla Fisica e dalla Metafisica di Aristotele, verrà denominata 'motrice', 'produttiva' o, più esplicitamente ancora, 'efficiente', non solo in quanto sono esplicitamente in gioco in questo passo procedimenti

PLATO, The electronic Journal of the International Plato Society, n 8, 2008.

http://gramata.univ-paris1.fr/Plato

(c) All rights of reproduction of any form reserved. 
generativi e produttivi, ma anche perché tale principio di causalità viene introdotto per giustificare, nel seguito immediato, l'individuazione di una tecnica, appunto, 'produttiva' (219b11-12), ossia di una competenza o di un sapere nell'ambito del quale 'tutte le cose che proprio adesso elencavamo' (219b8-9) l'agente produttore che conduce qualcosa all'essere e il prodotto che è condotto

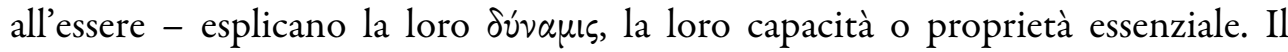

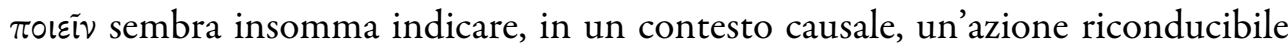
proprio alla causa efficiente.

Ammettendo, lo ribadisco nuovamente, come semplice ipotesi di lavoro, questi argomenti e queste conclusioni, si può rilevare che i sostenitori della causalità efficiente delle idee platoniche si trovano però a fronteggiare alcune temibili difficoltà. Questo articolo intende illustrare, in forma generale e sintetica, le due difficoltà che mi sembrano più impegnative dal punto di vista filosofico, relativamente $(\mathrm{A})$ al principio di 'chiusura causale della fisica' e (B) alla possibilità di un ordine razionale nel mondo, ossia alla legittimità di un finalismo naturale in una prospettiva teleologica orientata al bene.

Per quanto riguarda (A), il principio di 'chiusura causale della fisica', pressoché unanimemente condiviso nella contemporanea filosofia della fisica, prevede che qualunque causa che susciti un effetto di carattere fisico o comunque appartenente al mondo fisico deve essa stessa appartenere al mondo fisico ed essere di carattere fisico, e ciò nella misura in cui occorre ammettere una contiguità spazio-temporale, nel processo causale, fra la causa e il suo effetto (4). ̇̀ piuttosto chiaro che un principio di questo genere, applicato alle idee platoniche nell'esercizio della loro funzione causale in relazione alle cose sensibili, comporterebbe alcune conseguenze assai problematiche, perché imporrebbe alle idee, in quanto cause efficienti delle cose sensibili, una contiguità spaziotemporale rispetto alle cose sensibili, così violando il loro statuto di enti separati ed estranei all'universo sensibile spazio-temporale. Si porrebbe insomma un evidente conflitto fra lo statuto che alle idee appartiene per definizione e la funzione causale che a esse si deve attribuire, se tale causalità si rivela di natura propriamente attiva o produttiva o, più esplicitamente ancora, efficiente. Si può naturalmente dubitare che il principio di 'chiusura causale della fisica', come

PLATO, The electronic Journal of the International Plato Society, n 8, 2008.

http://gramata.univ-paris1.fr/Plato

(c) All rights of reproduction of any form reserved. 
sopra enunciato, sia stato condiviso da Platone e supporre perciò che, per Platone, una causa intellegibile, assolutamente differente dall'universo sensibile e separata da questo, estranea allo spazio e al tempo, possa senza difficoltà suscitare una serie ampia e varia di effetti sul piano fisico della realtà sensibile spazio-temporale, dunque senza stabilire nessuna forma di contiguità o di continuità, diretta $o$ indiretta, forte o debole, fra essere e divenire, fra intellegibile e sensibile, fra idee e cose.

Tuttavia, senza attardarsi adesso su un esame dettagliato dei passi pertinenti dei dialoghi, è piuttosto plausibile che le cose stiano diversamente. È sufficiente considerare le pagine centrali del Fedone, da 100d a 105b, per stabilire l'unico assunto, sul quale credo non sussistano dubbi interpretativi possibili, secondo cui ogni idea causa la proprietà di cui è l'idea e non quella contraria: il bello, per esempio, la bellezza e non la bruttezza, il caldo il calore e non il freddo, il grande la grandezza e non la piccolezza, sicché bellezza, calore e grandezza non hanno altre cause che il bello, il caldo e il grande e non certo il non bello (diverso o contrario del bello), il non caldo (diverso o contrario del caldo) e il non grande (diverso o contrario del grande). Tutto ciò permette di associare due ulteriori corollari all'assunto che regola la teoria platonica della causalità eidetica; bisogna ammettere: (a) una forma di omogeneità fra causa ed effetto, in modo che, pur ponendosi la proprietà ideale intellegibile (per esempio la bellezza in sé) e quella materiale sensibile (per esempio la bellezza fisica di un volto) su piani di realtà diversi (l'intellegibile e il sensibile, l'immateriale e il materiale), se la proprietà sensibile si trova a essere effettivamente causata dall'idea, nel senso che è propriamente prodotta o suscitata da essa e dipende perciò per la sua sussistenza dall'azione causale di quella, è necessario che sia la stessa proprietà - prima in forma intellegibile poi in forma sensibile - a essere trasmessa dalla causa che la suscita all'effetto che la accoglie; (b) inoltre, se è la stessa proprietà a sussistere prima in forma intellegibile poi in forma sensibile - come proprietà dell'idea che la suscita e la produce e come proprietà della cosa sensibile che la accoglie, in quanto l'azione causale avviene per omogeneità fra causa ed effetto, la causa risulta in qualche senso immanente nell'effetto, perché è la stessa proprietà intellegibile, intesa come idea in sé, a rendersi presente, in forma sensibile, nella cosa che la riceve (5). Del resto, (a) omogeneità fra causa ed effetto e (b)

PLATO, The electronic Journal of the International Plato Society, n 8, 2008.

http://gramata.univ-paris1.fr/Plato

(c) All rights of reproduction of any form reserved. 
FronterotTA, Francesco, "Chiusura causale della fisica e razionalità del tutto : alcune opzioni esegetiche sull'efficienza causale delle idee platoniche"

immanenza della causa nell'effetto non sono solo paradossali conseguenze derivanti da una distorta concezione della causalità impropriamente attribuita a Platone, perché i dialoghi forniscono un'importante testimonianza in tal senso: il Fedone (100d-e; ma molti altri passi potrebbero essere citati: cfr. per esempio Hipp. ma. 289d2-8; 292c9-d3; 300a9-b2; Euthyphr. 5d1-2; Phaed. 104b6-c1; 104e7-105a5; Symp. 211b2-5) collega la causalità delle idee rispetto al sensibile alla loro presenza nel sensibile o alla loro connessione con il sensibile ( $\varepsilon i \iota \varepsilon$

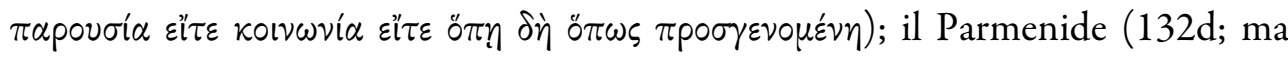
molti altri passi di altri dialoghi potrebbero essere citati: cfr. per esempio Euthyphr. 6e3-6; Phaed. 76d7-e4; Rep. X 597a1-5; Phaedr. 250a6-b5; 250e1251a7) propone una lettura della relazione causale fra idee e cose nella forma di un processo di assimilazione ( $(\dot{i} \kappa \alpha \sigma \theta \tilde{\eta} v a l):$ le idee esercitano la propria causalità rispetto alle cose rendendole in qualche misura simili a se stesse. Azione causale secondo omogeneità e immanenza della causa nell'effetto o, per esprimersi in termini platonici, assimilazione di cause ed effetti e presenza delle prime nei secondi, che rappresentano gli elementi costituenti del principio di 'chiusura causale della fisica', appaiono ben attestati nei dialoghi. Le idee-cause saranno allora, come vuole tale principio, contigue alle cose sensibili causate dal punto di vista spazio-temporale, sacrificando così la loro assoluta differenza ontologica, il loro statuto di enti eterni e separati dall'universo fisico?

Di fronte a un'alternativa così delicata e radicale (del resto prospettata già in Parm. 132c: come potrebbe l'idea-causa rimanere $\alpha \dot{\tau} \tau \grave{\eta} \kappa \alpha \theta^{\prime} \alpha \dot{\tau} \tau \eta^{\prime} \nu$, se è घे $\dot{\eta} \mu i \tau$ ?) mi sembra che si presentino tre opzioni esegetiche, tutte dipendenti dall'interpretazione di alcuni dialoghi tardi, come il Filebo e il Timeo, in cui Platone pare confrontarsi nel modo più serrato con questa difficoltà.

(A.a) Secondo un'interpretazione resa tradizionale dagli studi sul Timeo di Luc Brisson, che su questo punto rielabora alcune ipotesi abbozzate da Cornford e Cherniss, la soluzione elaborata dal tardo Platone consisterebbe nell'introduzione di un agente intermedio fra l'intellegibile e il sensibile cui è affidato il compito di compiere propriamente l'azione causale, di porre cioè operativamente in atto la causalità efficiente e produttiva che caratterizza la relazione fra le idee e le cose: l'ovvio riferimento, in questo contesto, è al demiurgo del Timeo (6), ma anche,

PLATO, The electronic Journal of the International Plato Society, n 8, 2008.

http://gramata.univ-paris1.fr/Plato

(c) All rights of reproduction of any form reserved. 


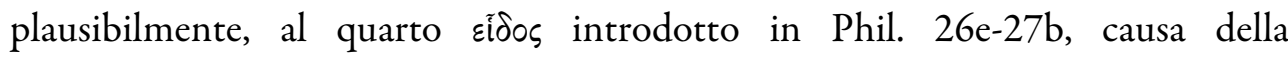

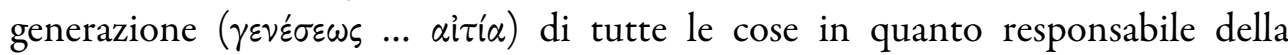

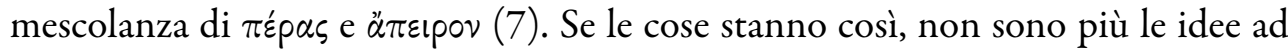
agire sulle cose sensibili come loro cause efficienti, ma appunto un agente intermedio che, servendosi delle idee come di modelli eterni e separati dall'universo fisico spazio-temporale, ne riproduce l'ordine e la disposizione nel sensibile, senza che ciò susciti nessuna contraddizione: in quanto $\pi \alpha p \alpha \delta \varepsilon i ́ \gamma \mu \alpha \tau \alpha$ privi di funzione attiva, le idee non corrono in tal caso nessun rischio di rimanere invischiate, a causa del principio di 'chiusura causale della fisica', nel mondo sensibile spazio-temporale, perché a subire questo esito sarà l'agente causale che, per definizione anfibio quanto alla sua costituzione e alla sua collocazione ontologica, risulterà a un tempo omogeneo all'intellegibile, di cui è in grado di apprendere l'essenza e di comprendere la natura per utilizzarle come modelli operativi nella sua azione causale, e al sensibile, in cui è in grado di operare concretamente producendo l'universo (8). Il problema che tuttavia non si può eludere, in questa prospettiva, riguarda direttamente la natura e lo statuto della causa demiurgica: va intesa 'letteralmente', dunque come una figura personale di cui spiegare le ragioni e le azioni, giustificare le decisioni e le attività, a cui attribuire volontà e personalità individuali? Oppure metaforicamente, come una funzione causale impersonale, di cui si deve supporre l'eternità e la continuità, ma anche verificare la consistenza razionale, in particolare chiedendosi, uscendo dalla metafora del mito cosmologico, come e dove si collochi nella struttura ontocosmologica della realtà? Mi pare si pongano in entrambi i casi una serie di difficoltà insormontabili.

(A.b) Prendendo le mosse dalle difficoltà appena enunciate, Franco Ferrari ha formulato una diversa proposta di lettura, basata su un'ampia serie di prove testuali e filosofiche, secondo la quale, in estrema sintesi, la divinità demiurgica altro non sarebbe che una metafora della funzione causale-efficiente che appartiene in effetti alle idee e che, dunque, non può essere dissociata dalle realtà intellegibili. Osservando che molti dei passi del Timeo in cui è introdotta la figura del demiurgo incoraggiano una comprensione simbolica dell'artigiano mitologico, Ferrari ipotizza che non solo Platone abbia inteso le idee come cause efficienti del mondo sensibile nei dialoghi precedenti, ma che, ancora in questo dialogo, egli

PLATO, The electronic Journal of the International Plato Society, n 8, 2008.

http://gramata.univ-paris1.fr/Plato

(c) All rights of reproduction of any form reserved. 
persista nella difesa di questa concezione dell'intellegibile (9). Senza entrare nel dettaglio di un esame di questa proposta (10) - si può comunque rilevare che, se essa ha una certa plausibilità per quanto riguarda l'interpretazione del Timeo, non pare invece molto soddisfacente per la comprensione del Filebo, in cui la

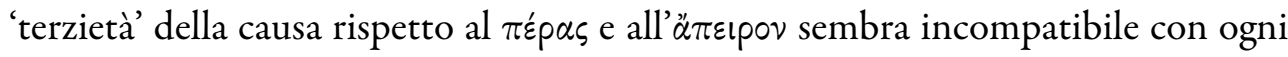
sua riduzione metaforica -, occorre però constatare che, rispetto alla difficoltà segnalata in relazione al principio di 'chiusura causale della fisica', un'ipotesi del genere non risolve (o piuttosto, forse, non si propone affatto di affrontare) la questione, lasciandola perciò semplicemente insoluta, senza precisare se ciò avvenga perché Ferrari non crede, contrariamente a quanto ho tentato di argomentare qui, nell'efficacia di tale principio o nella sua presenza in Platone, oppure perché ritiene che si tratti di una difficoltà in qualche modo, che egli stesso lascia imprecisato e che a me rimane oscuro, superabile.

(A.c) Resta un'ultima opzione esegetica, a mia conoscenza precedentemente inesplorata e che ho cercato di articolare in alcuni lavori recenti (11), secondo la quale bisogna ammettere che Platone abbia introdotto una realtà terza, intermedia e mediatrice fra l'intellegibile e il sensibile, che non funge però di per sé da agente causale efficiente, ma piuttosto da 'forza di interposizione' per evitare il contatto o la contiguità fra l'intellegibile e il sensibile, tuttavia traducendo la causalità metafisica delle idee, da esse esercitata direttamente, in una causalità fisica che sucita i propri effetti nel sensibile. Facendo riferimento al meccanismo che illustra le attitudini conoscitive dell'anima del mondo nel Timeo, ho tentato di spiegare come essa riesca a riprodurre il perfetto ordine formale delle idee sul piano sensibile e perché questa riproduzione assuma i tratti, sul piano sensibile, di una disposizione di tipo geometrico-matematico. Come è noto, l'anima del mondo è costituita a partire da una mescolanza di essere, identico e diverso, non però delle idee intellegibili dell'essere, dell'identico e del diverso, né delle corrispondenti realtà sensibili, ma di un misto di entrambe; l'unione di essere, identico e diverso messa in atto per dare forma all'anima del mondo non è affatto casuale, giacché segue una precisa disposizione matematica, stabilendo rigorose proporzioni numeriche per la combinazione dei tre elementi. Una volta realizzata questa mescolanza, il materiale ottenuto viene diviso in due strisce uguali, prima sovrapposte in forma di X, poi curvate per far coincidere le estremità delle due

PLATO, The electronic Journal of the International Plato Society, n 8, 2008.

http://gramata.univ-paris1.fr/Plato

(c) All rights of reproduction of any form reserved. 
strisce, ottenendo quindi due cerchi concentrici, l'uno inclinato rispetto all'altro come l'equatore rispetto all'eclittica, che si intersecano in due punti opposti: il primo, il cerchio dell'identico, ruota esternamente all'altro, il cerchio del diverso (34a-36b); il movimento del cerchio dell'identico è perfettamente regolare e uniforme, unico e indiviso, mentre quello del cerchio del diverso, pur regolare e uniforme, è invece diviso per sei volte, dando luogo perciò a sette traiettorie circolari concentriche, a loro volta caratterizzate da movimenti diversi (36d). Quando realizza la conoscenza attraverso il movimento del suo cerchio dell'identico, l'anima del mondo conosce davvero gli oggetti intellegibili cui il cerchio dell'identico sempre si rivolge e con cui si trova in stretta relazione; quando invece la realizza attraverso il movimento del suo cerchio del diverso, l'anima del mondo opina soltanto, e percepisce, gli oggetti sensibili cui il cerchio del diverso sempre si rivolge e con cui si trova in stretta relazione (37a-c). Il cerchio dell'identico produce intellezione e scienza ( quanto cerchio dell'identico, è l'identità che esso assicura fra il soggetto conoscente, l'anima, e l'oggetto conosciuto, l'intellegibile, a garantire la verità assoluta e immediata della conoscenza ottenuta, mentre il cerchio del diverso

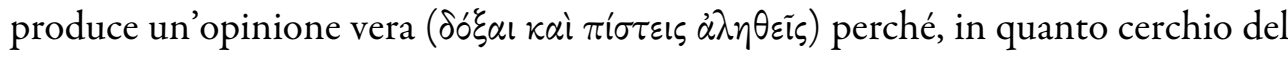
diverso, è l'alterità che esso introduce fra il soggetto conoscente, ancora una volta l'anima, e l'oggetto conosciuto, il sensibile, a determinare così quel giudizio discorsivo in cui consiste l'opinione. Ecco allora la domanda di partenza: cosa avviene quando il cerchio dell'identico 'tocca' un'idea? Questo contatto, per quanto non sensibile e non materiale e dunque, in qualche senso, metaforico, deve essere sufficientemente reale da produrre quella che chiamiamo conoscenza o intellezione, che è un'affezione dell'anima, una sua passione, dunque, in qualche senso, una sua modificazione. Ma il cerchio dell'identico consiste di una traiettoria circolare che, in virtù del suo movimento di rotazione uniforme, fa muovere il cerchio del diverso e, per suo tramite, gli altri sei cerchi concentrici che esso governa (36d). La trasmissione di questo movimento avviene naturalmente anch'essa per contatto, giacché il cerchio dell'identico e il cerchio del diverso, che il demiurgo ha disteso in due bande sovrapposte in un punto e che ha quindi piegato in forma di $\mathrm{X}$ facendone poi coincidere le estremità (36b-c), si intersecano perciò in due punti ed è verosimilmente proprio attraverso questi due punti di intersezione che si verifica la trasmissione del movimento; di seguito, il

PLATO, The electronic Journal of the International Plato Society, n 8, 2008.

http://gramata.univ-paris1.fr/Plato

(c) All rights of reproduction of any form reserved. 
cerchio del diverso, abbracciando e accompagnando con il suo movimento circolare quello dei sei cerchi concentrici che comprende sotto di sé, trasmette loro a sua volta tale movimento, che infine si propaga, via via degradando attraverso i cerchi inferiori e più interni, fino al centro dell'anima, che è congiunto al centro del corpo del mondo; ed ecco come il movimento dell'anima, prendendo avvio dal movimento perfettamente regolare e uniforme del cerchio dell'identico discende progressivamente, moltiplicandosi e diversificandosi, e, raggiunto infine il corpo, può così effettivamente animarlo. Ma in questa cinghia di trasmissione, cosa viene effettivamente trasmesso? Senza dubbio movimento, nei modi che abbiamo appena illustrato, ma non soltanto questo: quando infatti il cerchio dell'identico 'tocca' un'idea, ne subisce il contatto, che lascia in esso un'impronta dell'idea 'toccata', e ne è dunque modificato; il cerchio dell'identico prosegue quindi la sua traiettoria perfettamente regolare e uniforme, conservando però, nel punto in cui ha 'urtato' l'idea, una modificazione che appunto risulta dalla forma essenziale che l'idea ha impresso su di esso: non si tratta certo di una figura in senso proprio, perché non vi è una materia in senso proprio che possa accoglierne i tratti, ma, poiché il cerchio dell'identico, come del resto l'intera anima, ha una struttura e una configurazione geometrico-matematica, la modificazione prodotta dal contatto con l'idea avrà l'aspetto di una modificazione di carattere geometrico-matematico, supponendo per esempio che la circonferenza del cerchio dell'identico subisca nel punto in cui si è verificato il contatto con l'idea una variazione numerica o un'oscillazione quantitativa dei suoi elementi componenti. Ora, proseguendo nella sua rotazione, il cerchio dell'identico interseca il cerchio del diverso in due punti dati, in ciascuno dei quali, nel corso della rotazione, si troverà a passare anche la modificazione subita dal cerchio dell'identico per il contatto con l'idea. Vale a dire che il cerchio dell'identico, oltre a trasmettere al cerchio del diverso il movimento regolare e uniforme che lo caratterizza, imprime anche in esso la modificazione che aveva subito per il contatto con l'idea e che conserva impressa - quindi trasmette in effetti una traiettoria circolare che reca in un suo punto una modificazione corrispondente alla forma essenziale dell'idea 'toccata' dal cerchio dell'identico, ossia alla variazione numerica o all'oscillazione quantitativa dei suoi componenti che in quel punto si è verificata. Non è difficile immaginare il seguito di questo processo di trasmissione attraverso i cerchi concentrici tracciati all'interno del

PLATO, The electronic Journal of the International Plato Society, n 8, 2008.

http://gramata.univ-paris1.fr/Plato

(c) All rights of reproduction of any form reserved. 
cerchio del diverso, poi, in virtù del contatto fra anima e corpo del mondo stabilito nella coincidenza dei rispettivi centri, fin nel corpo del mondo, sicché si tratterà non solo dell'estensione o dell'irraggiamento di una traiettoria e di un movimento, ma anche della modificazione di tale traiettoria che assume l'aspetto di una variazione numerica o di un'oscillazione quantitativa che, impressa infine su una materia sensibile, appunto sul corpo del mondo, si tradurrà in una figura concreta e visibile, derivante da una particolare distribuzione degli elementi geometrici che compongono il corpo di tutte le cose e dalla loro conseguente disposizione spaziale. Applicando questo modello esplicativo su vasta scala, esteso alla totalità delle idee 'toccate' dall'anima del mondo, conosciute in parte simultaneamente, in virtù di un'attitudine verosimilmente sinottica dell'anima del mondo, in parte in una successione temporale, sarà possibile giustificare la complessa disposizione dell'universo, la sua condizione stabile ed eterna e a un tempo, sotto altri rispetti, mutevole e diveniente; come pure sarà possibile spiegare ragioni e modalità per cui e in cui l'ordine puramente formale dell'intellegibile si traduce, nel sensibile, in un ordine quantitativo di carattere geometrico-matematico.

Se le cose stanno davvero così, potremo riconoscere che, in un senso, le idee rimangono davvero le cause efficienti o produttive del sensibile (come, lo si è visto poco sopra, vuole Ferrari), e questo nella misura in cui esse sono ciò che viene propriamente causato o prodotto nell'effetto, cioè il contenuto proprio dell'azione causale, ma è l'anima del mondo a esercitare la necessaria azione motrice compiendo così concretamente la trasmissione dell'effetto causato, ossia 'attuando' l'azione causale: l'idea è dunque causa efficiente o produttiva solo nel senso che rappresenta il contenuto trasmesso o, appunto, 'causato' nell'azione causale, mentre l'anima è l'ente che esercita tale azione, traducendo la causa intellegibile in un effetto sensibile. Sarà dunque l'anima a rimanere imprigionata, in virtù del principio di 'chiusura causale della fisica', nel mondo sensibile, allo stesso tempo però, in ragione della sua duplice costituzione e della sua collocazione ontologica intermedia, restando in contatto con l'intellegibile. La causazione, che richiede un contatto fra la causa ed l'effetto (o l'immanenza della prima nel secondo) e una forma di movimento nel processo che dalla causa conduce all'effetto, può essere compiuta esclusivamente dall'anima del mondo, il

PLATO, The electronic Journal of the International Plato Society, n 8, 2008.

http://gramata.univ-paris1.fr/Plato

(c) All rights of reproduction of any form reserved. 
solo ente che di per sé si configuri come un principio di movimento e che possieda una natura mista, capace di stabilire un contatto o una contiguità tanto con il sensibile quanto con l'intellegibile: ora, poiché questo ente è costituito in base a proporzioni numerico-matematiche, le modalità attraverso cui esso traduce la causalità intellegibile in un effetto sensibile saranno a loro volta di carattere numerico-matematico, sicché, concludendo con un esempio, ciò che il bello in sé è nell'intellegibile diviene nel sensibile, per azione dell'anima del mondo, una certa ordinata e armoniosa disposizione di elementi materiali (12).

Per quanto riguarda (B), la seconda difficoltà che mi limito a illustrare qui rispetto all'attribuzione di una causalità efficiente alle idee platoniche è relativa al riconoscimento di un ordine razionale e finalistico nel mondo e si può formulare come segue: se le idee sono le cause efficienti e produttive del mondo sensibile, nel senso che alla loro azione (eventualmente mediata da un agente causale intermedio) bisogna ricondurre, totalmente e senza residui, l'ordine e la disposizione della realtà fisica, 'perché' le idee operano come effettivamente fanno nel mondo sensibile - tale 'perché' dovendosi intendere così: per quale ragione o, più esattamente, in base a quale presupposto o principio il mondo sensibile appare ordinato e disposto secondo una logica complessiva che pare dipendere da un progetto intelligente, perseguire un fine, obbedire a leggi e criteri determinati e determinabili in un quadro razionale? Tre possibili risposte si presentano ancora una volta a queste domande.

(B.a) Si può ipotizzare l'esistenza di un voṽ sterzo, oltre le idee e le cose sensibili, capace di utilizzare i modelli ideali, quasi combinandoli in una sorta di mosaico o di puzzle cosmico, nella produzione e nella disposizione del mondo sensibile. In tal caso, l'introduzione di una divinità demiurgica, dotata di caratteri individuali, se non di personalità, e di capacità di raziocinio, di previsione e di calcolo, garantisce razionalità e finalità dell'ordine e della disposizione del cosmo. Questa ipotesi, evidentemente connessa a un'interpretazione letterale del mito cosmologico del Timeo, va però incontro, a mio avviso, alle stesse difficoltà sollevate sopra, in (A.a), relativamente alla necessità e alla possibilità di spiegare in modo soddisfacente la comparsa improvvisa e per molti aspetti ingiustificata di questa sorta di 'deus-ex-machina' che viene evocato, e 'miracolosamente' compare,

PLATO, The electronic Journal of the International Plato Society, n 8, 2008.

http://gramata.univ-paris1.fr/Plato

(c) All rights of reproduction of any form reserved. 
come causa prima e garanzia ultima del buon equilibrio dell'universo. Comunque sia di queste difficoltà, la progressiva emergenza e supremazia della figura del demiurgo nella vicenda del cosmo è un tratto significativamente presente nella storia delle interpretazioni, specie medioplatoniche, del Timeo.

(B.b) Una seconda risposta possibile all'interrogativo di partenza consiste nel

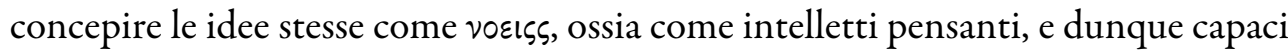
di porre direttamente in atto, nell'esercizio della loro azione causale, un disegno o un progetto razionale e intelligente, articolandone lo svolgimento e prefissandogli dei fini. D questo esito, in una certa misura adottato da alcuni esponenti della tradizione neoplatonica, e particolarmente da Plotino (cfr. per esempio Enn. V 9

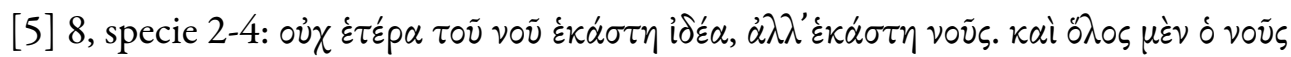

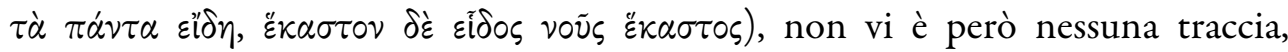
esplicita o implicita, nei dialoghi di Platone né delle testimonianze relative alla sua dottrina. Va inoltre ricordato che, anche quando Plotino stabilisce una coincidenza fra idee intellegibili e intelletti pensanti, ciò non lo induce in nessun caso a considerare tali 'idee-intelletti' come principi dotati riflessione, nel senso di una capacità di ragionamento o previsione, giacché, anzi, egli ribadisce con forza che nell'eternità e nella simultaneità dell'intellegibile non sono ammissibili né previsione né ragionamento, potendosi al massimo concedere che tutte le cose sono «tali quali un uomo sapiente le avrebbe previste» (Enn. VI 7 [38] 1, 29-32), vale a dire che solo dal nostro punto di vista inferiore e posteriore il mondo appare costituito secondo un piano razionale e provvidenziale caratterizzato da fini, senza che, tuttavia, tale condizione corrisponda alla realtà oggettiva dell'effettiva genesi del mondo. In altre parole, Plotino preferisce confinare ogni concezione razionale e provvidenziale del mondo nell'ambito delle valutazioni che degli esseri dotati di una visione solo parziale e condizionata delle cose possono avere, percependo come insuperabile la difficoltà di comprendere e giustificare la comparsa di un soggetto raziocinante, di qualunque natura, o piuttosto di un pensiero soggettivo come origine e fonte della razionalità del tutto, che si tratti di un demiurgo singolo e responsabile o di una molteplicità di soggetti pensanti, come una sorta di organizzazione solidale di principi contemporaneamente, ma non concorrenzialmente, attivi sul sensibile. I

PLATO, The electronic Journal of the International Plato Society, n 8, 2008. 
FronterotTA, Francesco, "Chiusura causale della fisica e razionalità del tutto : alcune opzioni esegetiche sull'efficienza causale delle idee platoniche"

paradossi impliciti in una simile prospettiva non hanno bisogno, credo, di essere sottolineati ulteriormente.

(B.c) Assumendo in parte la posizione di Plotino appena tratteggiata, la terza e più radicale soluzione del dilemma consiste nel suo puro e semplice scioglimento: alla domanda relativa al fondamento e alle ragioni che presiedono all'azione causale delle idee, bisogna rispondere che le idee non seguono né pensiero né logica, sicché la loro azione causale si rivela puramente meccanica e ciecamente necessaria, dipendendo solo, altrettanto meccanicamente e necessariamente, dalla natura stessa delle idee. Siamo dunque noi a riconoscere ragione e logica, ordine e finalità, nell'opera realizzata in seguito all'azione causale delle idee, senza però che nulla di tutto ciò si trovi già nell'azione causale stessa; e siamo ancora e soltanto noi a sollevare una tale richiesta di senso. L'unica risposta sensata a questa domanda è che, se l'azione causale delle idee fosse diversa da come è, non potremmo riconoscervi ragione e logica, ordine e finalità, né, forse, esisteremmo noi stessi come esseri capaci di porci tale domanda. Ma è allora la domanda stessa a non avere senso di per sé, in quanto essa può essere posta soltanto a posteriori, cioè nella misura in cui le cose sono come sono.

Si sarà compreso che, personalmente, opto in entrambi i casi per la terza ipotesi interpretativa come risposta alle difficoltà $(\mathrm{A})$ e (B), vale a dire per la (A.c) e la (B.c).

Francesco Fronterotta

Università del Salento (Lecce, Italy)

francesco.fronterotta@ateneo.unile.it

PLATO, The electronic Journal of the International Plato Society, n 8, 2008.

http://gramata.univ-paris1.fr/Plato

(c) All rights of reproduction of any form reserved. 


\section{NOTE}

(1) Occorre precisare che, parlando di causalità 'formale' e di causalità 'efficiente' o 'produttiva' in riferimento alle idee platoniche, non alludo necessariamente, pur mutuandone il linguaggio, alle corrispondenti categorie causali aristoteliche: intendo semplicemente (1) la causalità 'efficiente' eventualmente attribuita alle idee come una forma di causalità 'produttiva', cioè tale che le idee hanno il potere di suscitare, in ciò che ne partecipa, la proprietà di cui sono le idee, sicché attraverso la partecipazione si determina effettivamente il passaggio o la trasmissione di una certa proprietà da un ente che la possiede, l'idea-causa, a un ente che non la possiede, la cosa partecipante; come intendo (2) la causalità 'formale' delle idee come una forma di causalità 'logico-metafisica', cioè tale che le idee permettono di riconoscere, in ciò che ne partecipa, la proprietà di cui sono le idee, sicché attraverso la partecipazione non si determina effettivamente il passaggio o la trasmissione di una certa proprietà da un ente che la possiede, l'idea-causa, a un ente che non la possiede, la cosa partecipante, ma soltanto il riconoscimento e la denominazione di tale proprietà, già di per sé in possesso delle cose partecipanti. Si veda in proposito, Vlastos, G., 'Reasons and causes in the Phaedo', originariamente edito in Philosophical Review 78 (1969), 291-325. Fra gli studi che hanno contribuito a superare l'impostazione di Vlastos vanno ricordati particolarmente Fine, G., 'Forms as causes, Plato and Aristotle', [1987], ora in Fine, G., Plato on knowledge and forms. Selected essays, Oxford, 2003, 350-96; Sedley, D., 'Platonic causes', Phronesis 43 (1998), 114-32; Hankinson, R.J., Cause and explanation in ancient Greek thought, Oxford, 1998, 84-124. Più espliciti nell'attribuzione alle idee di una causalità efficiente sono invece Natali, C., 'La forma platonica è una causa formale?', in Platon und Aristoteles - sub ratione veritatis. Festschrift für W. Wieland zum 70. Geburtstag, hrsg. v. Damschen, G., Enskat, R. \& Vigo, A., Göttingen, 2003, 158-73, e Ferrari, F., 'Questioni eidetiche', Elenchos 24 2003, 93-113. A parte vanno considerati gli studi successivi di David Wolfsdorf sul problema dello statuto e della funzione delle idee in alcuni dialoghi platonici: 'Euthyphro 10a2-11b1: A study in Platonic

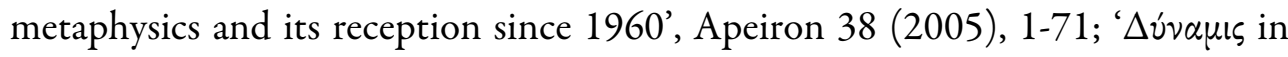
Laches', Phoenix 59 (2005), 324-47; 'Hippias Major 301b2-c2: Plato's critique of corporeal conception of Forms and of the Form-Participant relation', Apeiron 39

PLATO, The electronic Journal of the International Plato Society, n 8, 2008.

http://gramata.univ-paris1.fr/Plato

(c) All rights of reproduction of any form reserved. 
FronterotTA, Francesco, "Chiusura causale della fisica e razionalità del tutto : alcune opzioni esegetiche sull'efficienza causale delle idee platoniche"

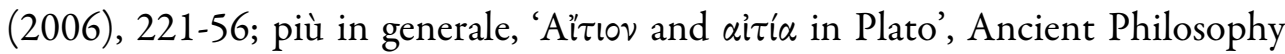
25 (2005), 341-48.

(2) Si veda in generale il mio studio $M \dot{\varepsilon} \theta \varepsilon \xi 1 \zeta$. La teoria platonica delle idee e la partecipazione delle cose empiriche. Dai dialoghi giovanili al Parmenide, Pisa, 2001, 195-222 e 381-95. Più nel dettaglio, della nozione di causa e della funzione causale nei dialoghi considerati giovanili mi sono occupato nell'Annexe III a Platon, Hippas majeur \& Hippias mineur, présentations et traductions par Pradeau, J.-F. et Fronterotta, F., Paris, 2005, 231-42, e nell'articolo 'The development of Plato's theory of ideas and the 'Socratic question", Oxford Studies in Ancient Philosophy 32 (2007), 37-62; ai dialoghi considerati della maturità di Platone e fino al Parmenide, ho dedicato l'articolo 'Il problema della

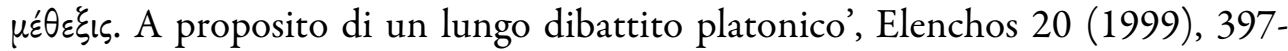
415, e il volume Guida alla lettura del Parmenide, Roma-Bari, 1998, specie 25-83; al Timeo e al Filebo, l'Introduzione a Platone, Timeo, a cura di Fronterotta, F., Milano, 2006², 30-70, l'articolo 'Questioni eidetiche in Platone: il sensibile e il demiurgo, l'essere e il bene', Giornale critico della filosofia italiana 85 (2006), 412-36, 412-24 e 431-36, e il saggio, di prossima pubblicazione, Nature and structure of the cause in Philebus 26e1-27b3.

(3) $\mathrm{Su}$ questo aspetto ho proposto alcune riflessioni nel mio articolo 'L'interprétation néo-kantienne de la théorie platonicienne des idées et son 'héritage' philosophique', Revue philosophique de Louvain 98 (2000/2), 318-40.

(4) Si veda in proposito Kim, J., Physicalism, or something near enough, Princeton, 2005, 13-21, specie 15-17.

(5) Mi pare sufficientemente chiaro da quanto detto che l'omogeneità cui faccio qui riferimento non è di carattere numerico, tale cioè che causa ed effetto si riducono a un'unica entità, perché anzi, per essere tali, essi devono rimanere distinti; non è neanche, almeno a priori, un'omogeneità di carattere ontologico, tale cioè che causa ed effetto sussistono sullo stesso piano come enti dotati dello stesso statuto ontologico, perché le idee intelligibili e le cose sensibili si trovano per definizione, secondo Platone, su piani di realtà distinti e separati; non è infine

PLATO, The electronic Journal of the International Plato Society, n 8, 2008.

http://gramata.univ-paris1.fr/Plato

(c) All rights of reproduction of any form reserved. 
un'omogeneità specifica, tale cioè che causa ed effetto possiedono la stessa identità, così riducendosi a uno stesso ente. Si tratta invece di una particolare forma di omogeneità generica, tale cioè che, pur sussistendo causa ed effetto come enti distinti per numero, identità e statuto ontologico, la proprietà o il contenuto trasmessi nell'azione causale dalla causa, e ricevuti come effetti in esso suscitati dal soggetto che subisce tale azione, coincidono con la causa stessa, che di conseguenza si rivela della stessa natura dell'effetto suscitato, stabilendo perciò con esso una relazione di continuità o di contiguità che implica una qualche forma di immanenza nell'effetto: se la proprietà o il contenuto dell'azione causale coincidono con la causa stessa, sarà la causa stessa, pur distinguendosi come ente dal soggetto che subisce l'azione causale, a essere in qualche modo presente in esso e a 'darsi' in qualche modo a esso come effetto. L'operatività della causa, insomma, coincide con la sua stessa natura causale, e ciò comporta che il risultato della sua azione produttiva sia omogeneo a essa, perché essa 'fa' ciò che 'è', e che essa sia immanente nel proprio prodotto, perché essa 'è' in ciò che 'fa'. Dunque, nel caso della relazione causale fra le idee e le cose sensibili, anche se vi è un'eterogeneità numerica, specifica e ontologica, deve sussistere fra esse un'omogeneità generica che dipende dalla trasmissione di una stessa proprietà $o$ di uno stesso contenuto causale dalle idee alle cose sensibili; e poiché l'idea che conferisce questa proprietà o questo contenuto causale alle cose sensibili è essa stessa questa proprietà o questo contenuto causale, ne segue che ciò che l'idea causa nella cosa sensibile e rende in essa presente è in qualche modo se stessa. Pur non potendo l'idea, ente immateriale e intellegibile, risultare concretamente o fisicamente immanente nelle cose sensibili partecipanti, essa deve però stabilire rispetto a esse, in virtù della sua funzione causale, una contiguità spazio-temporale che pare tuttavia in contrasto con la sua definizione di ente separato ed estraneo allo spazio e al tempo.

(6) Cfr. Brisson, L., Le même et l'autre dans la structure ontologique du Timée Platon. Un commentaire systématique du Timée de Platon, Sankt Augustin, 19942, 29-106, specie 85-86. Sul tema si vedano in precedenza Cornford, F.M., Plato's cosmology, The Timaeus of Plato translated with a running commentary, London, 1937, 34-39, e Cherniss, H.F., Aristotle's criticism of Plato and the

PLATO, The electronic Journal of the International Plato Society, n 8, 2008.

http://gramata.univ-paris1.fr/Plato

(c) All rights of reproduction of any form reserved. 
FronterotTA, Francesco, "Chiusura causale della fisica e razionalità del tutto : alcune opzioni esegetiche sull'efficienza causale delle idee platoniche"

Academy, Baltimore, 1944, App. XI. Ho a mia volta difeso questa intepretazione tradizionale nella mia introduzione a Platone, Timeo, cit., 51-70.

(7) Cfr. su questo punto Cherniss, H. F., 'Some war-time publications concerning Plato', American Journal of Philology 68 (1947), 225-34; Boussoulas, N.-I., L'être et la composition des mixtes dans le Philèbe de Platon, Paris, 1952, 175-77; e il mio articolo Nature and structure of the cause in Philebus 26e127b3, cit.

(8) Bisogna naturalmente distinguere fra la causa intelligente del Filebo, che opera facendo intervenire misura e proporzione nella mescolanza di $\pi \dot{\varepsilon}$ pas e

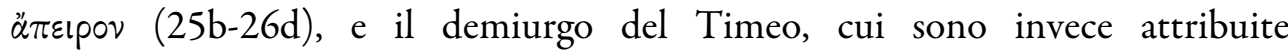
competenze artigianali che gli permettono di lavorare manualmente un materiale preesistente nella sua attività produttiva. È del resto verosimile che, al di là della metafora demiurgica, anche il Timeo intenda la mediazione causale che porta alla costituzione del mondo sensibile a partire dal modello intellegibile in termini matematici, cioè in virtù dell'applicazione al materiale sensibile di un ordine numerico o geometrico che ne garantisce la somiglianza rispetto al modello intellegibile.

(9) Cfr. Ferrari, F., 'Causa paradigmatica e causa efficiente: il ruolo delle idee nel Timeo', in Plato physicus. Cosmologia e antropologia nel Timeo, a cura di Natali, C. e Maso, S., Amsterdam, 2003, 83-96. Anticipazioni significative di un'interpretazione di questo genere si trovavano già negli studi di Halfwassen, J., 'Der Demiurg: seine Stellung in der Philosophie Platons und seine Deutung im antiken Platonismus', in Le Timée de Platon. Contribution à l'histoire de sa réception. Platos Timaios. Beiträge zu seiner Rezeptionsgeschichte, éd. par Neschke-Hentschke, A., Louvain-Paris, 2000, 39-62; e Dillon, J., 'The riddle of the Timaeus: is Plato sowing clues?', in Studies in Plato and the Platonic tradition, essays presentd to J. Whittaker, ed. by Loyal, M., Aldershot-BrookfieldSingapore-Sidney, 1997, 24-42.

(10) Cfr. Fronterotta, F., 'Questioni eidetiche in Platone: il sensibile e il demiurgo, l'essere e il bene', cit., 420-24.

PLATO, The electronic Journal of the International Plato Society, n 8, 2008.

http://gramata.univ-paris1.fr/Plato

(c) All rights of reproduction of any form reserved. 
FronterotTA, Francesco, "Chiusura causale della fisica e razionalità del tutto : alcune opzioni esegetiche sull'efficienza causale delle idee platoniche"

(11) Mi riferisco ai miei lavori 'Il Timeo e la matematica embodied', in La sapienza di Timeo, a cura di Napolitano, L., Milano, 2007, 173-206, e soprattutto 'Intelligible Forms, Mathematics, and the Soul's Circles: an Interpretation of Tim. 37a-c', Les Études platoniciennes 4 (2007), 119-127.

(12) Si potrebbe obiettare che, così concepita, la causalità delle idee finisce per coincidere con quella che Aristotele chiamerà causa formale, lasciando interamente all'anima del mondo l'esercizio della causalità motrice o efficiente. Una simile conclusione dipende in realtà dal modo in cui si intendono, nella dottrina aristotelica, la causa formale e la sua relazione con la causa efficiente: se la causa formale non è altro che 'la definizione di ciò in cui consiste l'essere' di qualcosa, 'i generi di essa' e 'le parti della definizione' (Phys. II 194b-195a), pare lecito concluderne che alle idee spetta, nel Timeo, una funzione assai più concreta ed efficace, giacché sono le idee stesse a intervenire, tramite l'anima del mondo che funge da agente intermediario, sul mondo sensibile, rappresentando propriamente i contenuti che, con la mediazione dell'anima, sono trasmessi dall'intellegibile (in cui sussistono come pure essenze formali) al sensibile (in cui assumono l'aspetto di una configurazione materiale ben precisa). Se invece, facendo specialmente riferimento alla causalità operante nella generazione naturale, intendiamo la causa motrice o efficiente di Aristotele come un semplice agente responsabile della trasmissione della forma, sarà proprio la forma a costituire il contenuto preesistente trasmesso nel processo causale, così adempiendo alla funzione di una causa, seppur definita formale, di fatto effettivamente produttrice.

PLATO, The electronic Journal of the International Plato Society, n 8, 2008. 